\title{
Modeling and Throughput Analysis for SMAC with a Finite Queue Capacity
}

\author{
Ou Yang and Wendi Heinzelman \\ Dept. of Electrical and Computer Engineering, University of Rochester \\ Rochester, NY, 14623, USA \\ oyang@ece.rochester.edu, wheinzeldece.rochester.edu
}

\begin{abstract}
SMAC is a popular duty-cycled MAC protocol, designed for wireless sensor networks to save energy and prolong the network lifetime. However, existing work evaluates the performance of SMAC solely through simulations or field measurements. To the best of our knowledge, there are no analytical models for evaluating the performance of SMAC. In this paper, we propose a Markov model to describe the behavior of SMAC with a finite queue capacity. This model enables us to find the expected throughput of SMAC under variable number of nodes, queue capacities, contention window sizes, and data arrival rates. We validate the model through extensive simulations, which provide throughput values within $5 \%$ of the throughput values obtained through our model. Our proposed Markov model can be used to estimate the throughput of SMAC under many different network and node conditions, and more importantly, it provides us with a better understanding of the way that different parameters affect the performance of SMAC.
\end{abstract}

\section{INTRODUCTION}

Wireless sensor networks have attracted much interest in both academia and industry due to their low cost, ease of deployment, and support for various applications ranging from military surveillance and emergency rescue to medical monitoring. However, energy constraints imposed by the battery-powered sensor nodes are a limiting factor, preventing the ubiquitous use of wireless sensor networks. Therefore, much research has focused on how to save energy and prolong the network lifetime [1][2]. In particular, a MAC protocol can put sensors to sleep periodically to reduce idle listening [2][4], which is energy intensive in wireless sensor networks. SMAC [2] was the first such duty-cycled MAC protocol designed for wireless sensor networks. It is also one of the most popular MAC protocols used for research on and implementation of wireless sensor networks [3][6]. However, most existing work evaluates the performance of SMAC via simulations or field measurements [2][5][6]. To the best of our knowledge, there is no analytical model for evaluating the performance of SMAC.

In this paper, we focus on throughput analysis of SMAC with a finite queue capacity. We propose two Markov models to describe the behavior of SMAC and to further calculate its throughput with and without retransmissions. We show that the throughput obtained from our analytical model matches simulation results under various scenarios. Our SMAC model can be used to estimate the throughput for a given SMAC configuration. Throughput estimation is important for many applications, like visual surveillance, which generate large amounts of data. Moreover, our model can be used to optimize the parameters of SMAC (e.g., queue capacity and contention window size) for a given network and traffic load so that the highest throughput can be reached for a given duty cycle (given power consumption). Furthermore, our model can be used to arbitrate the trade-off between throughput and network lifetime by choosing appropriate SMAC duty cycles. Since a smaller duty cycle leads to a longer network lifetime but lower throughput, for some applications that require minimum throughput or upper-layer protocols that have important signaling, an appropriate duty cycle can maximize the network lifetime while maintain a required quality of service.

The rest of the paper is organized as follows. Section II introduces the design of SMAC. Section III presents our Markov model for SMAC with and without retransmissions. Section IV shows the process of obtaining throughput from the proposed model. Section V validates our model and discusses the performance of SMAC. Section VI discusses the related work. Finally, Section VII concludes the paper.

\section{SMAC PROTOCOL}

To prolong the network lifetime, SMAC operates in a dutycycled fashion, i.e., sensors sleep and wake up periodically, as shown in Fig. 1. The active period of a cycle has a fixed length, which is determined by the MAC layer contention window size. The sleeping period of a cycle, instead, could be shorter or longer, depending on the predefined duty cycle, which is the ratio of the active period length to the cycle length. All the nodes in the network have the same cycle length and duty cycle. To improve the communication efficiency, SMAC synchronizes sensors by exchanging their sleep-awake schedules in SYNC packets. A fixed interval in each active period is reserved for SYNC packet exchange.

SMAC uses RTS/CTS/DATA/ACK to guarantee successful unicast transmissions. However, SMAC has a fixed contention window, as a change in contention window size changes the length of an active period and hence influences the synchronization process. Moreover, when a node fails to win the contention or it encounters an RTS collision, it goes to

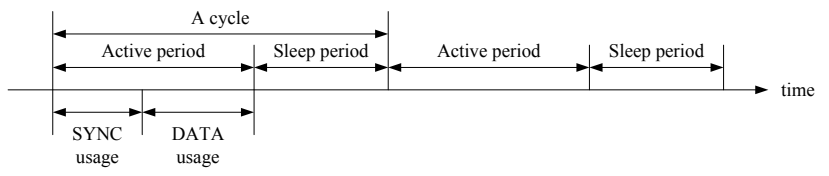

Fig. 1. Sleep-awake cycles of SMAC. 
sleep until the next active period. On the other hand, when a node sends out an RTS successfully, it does not go back to sleep until the transmitted DATA packet is acknowledged.

It is necessary to list all the reasons for DATA packet loss in SMAC. Assuming ideal channels (i.e., no hidden terminals, capture effect or fading), a DATA packet could be dropped due to (1) overflow of the DATA packet queue, (2) failure of the associated RTS if there is no retransmission, and (3) over the retransmission limit if retransmissions are supported.

\section{MARKOV MODEL OF SMAC}

We propose a Markov model to describe the behavior of SMAC at individual nodes. The model assumes that (1) each node has independent packet arrival, (2) each node has a finite FIFO queue, and (3) the channel is ideal. As a result, nodes can hear each other in the network, and they are well synchronized. The proposed Markov model has finite states, each of which represents a different status of a node at the wake-up instant of a cycle. For SMAC without retransmissions, the number of packets in the queue corresponds to different states in a 1-D Markov model. For SMAC with retransmissions, a 2-D Markov model is proposed, where each state has an index of queue length and an index of retransmission stage. A node may change status cycle by cycle, corresponding to the transition from one state to another in the Markov model. Table I lists the notations that are used throughout the paper.

\section{A. Modelling SMAC without Retransmissions}

Fig. 2 shows the 1-D Markov model of SMAC without retransmissions. This model has $Q+1$ states, from left to right corresponding to 0 packets in the queue to $Q$ packets in the queue (full queue). The transition probabilities from one state to another can be calculated as follows.

$$
\begin{aligned}
& P_{0, i}=A_{i}, i=0 . . Q-1 \\
& P_{0, Q}=A_{\geq Q} \\
& P_{i, i-1}=p \cdot A_{0}, i=1 . . Q \\
& P_{i, j}=p \cdot A_{j-i+1}+(1-p) \cdot A_{j-i}, i=1 . . Q-1, j=i . . Q-1 \\
& P_{i, Q}=p \cdot A_{\geq Q-i+1}+(1-p) \cdot A_{\geq Q-i}, i=1 . . Q \\
& P_{i, j}=0, i=2 . . Q, j=0 . . i-2
\end{aligned}
$$

Specifically, (1) and (2) describe the fact that the transition from an empty-queue state to a non-empty-queue state depends only on the new packet arrivals, $A_{i}$. Equations (3) and (6) describe the fact that a node can only transmit one DATA packer per cycle with a probability $p$, and the probability of having one packet less in the queue equals to

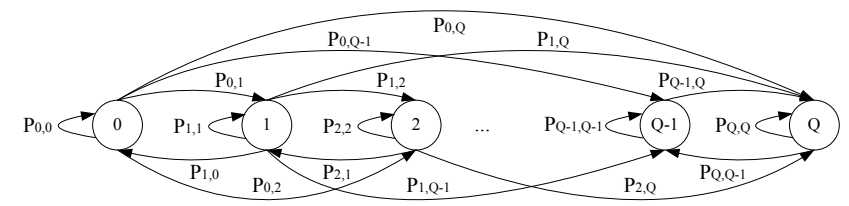

Fig. 2. 1-D Markov model for SMAC without retransmissions.
TABLE I. NOTATIONS

\begin{tabular}{|l|l|}
\hline \hline \multicolumn{1}{|c|}{ Symbol } & \multicolumn{1}{c|}{ Quantity } \\
\hline$N$ & number of nodes in the network \\
\hline$Q$ & queue capacity in units of a DATA packet \\
\hline$R$ & retransmission limit \\
\hline$d$ & duty cycle \\
\hline$W$ & length of a cycle \\
\hline$\lambda$ & contention window size in units of ms \\
\hline$A_{k}$ & $\begin{array}{l}\text { patA packet arrival rate at the MAC layer } \\
A_{k}=e^{-\lambda T}(\lambda T)^{k} / k ! \text { in the case of a Poisson arrival }\end{array}$ \\
\hline$A_{2}$ & $\begin{array}{l}\text { probability of no less than } k \text { DATA packets arriving in a } \\
\text { cycle }{ }_{2 k} \sum_{i=0} A_{i}\end{array}$ \\
\hline$p_{S}$ & probability of successful transmission of a DATA packet \\
\hline$p_{f}$ & probability of transmission failure of a DATA packet \\
\hline$p$ & probability of winning the contention $p=p_{s}+p_{f}$ \\
\hline$S$ & MAC layer DATA packet size \\
\hline$T H R^{S y s}$ & system throughput of SMAC \\
\hline \hline
\end{tabular}

the probability of winning the contention (sending an RTS) times the probability of no packet arrival in a cycle. Moreover, (4) and (5) describe the fact that the probability of having a non-decreasing queue can be divided into two parts depending on whether the oldest DATA packet in the queue wins the contention (first term) or not (second term). Finally, (2) and (5) show that packets are dropped when the queue overflows.

The proposed Markov model assumes that every node has a constant $p$ regardless of any node's queue lengths. Similar assumptions were made in [7][8], and were verified as good approximations of the real case. This conclusion is again confirmed through our model validation.

\section{B. Modelling SMAC with Retransmissions}

For SMAC with retransmissions, the retransmission stage as well as the queue length determines the status of a node. Specifically, when $R$ retransmissions are supported, there are $R+1$ retransmission stages to describe the retransmission status. Note that a node with an empty queue is always in retransmission stage 0. Fig. 3 shows the 2-D Markov model of SMAC with $R$ retransmissions and queue capacity $Q$. It has $Q \cdot(R+1)+1$ states, each of which is represented by two indices: retransmission stage and queue length.

We first look at the transitions from the empty-queue state. Their probabilities depend only on the new packet arrivals.

$P_{(0,0)->(0, i)}=A_{i}, i=0 . . Q-1$

$P_{(0,0)->(0, Q)}=A_{\geq Q}$

Then, we consider the transitions within one retransmission stage. Here, $p_{s}$ and $p_{f}$ are the probabilities of a successful and failed DATA packet transmission, respectively. Equations (9), (10) and (11) describe the transitions within retransmission stage 0 , whereas (12) and (13) describe the transitions within a non-zero retransmission stage.

$$
P_{(0, j)->(0, k)}=p_{s} \cdot A_{k-j+1}+(1-p) \cdot A_{k-j}, j=1 . . Q-1, k=j . . Q-1
$$




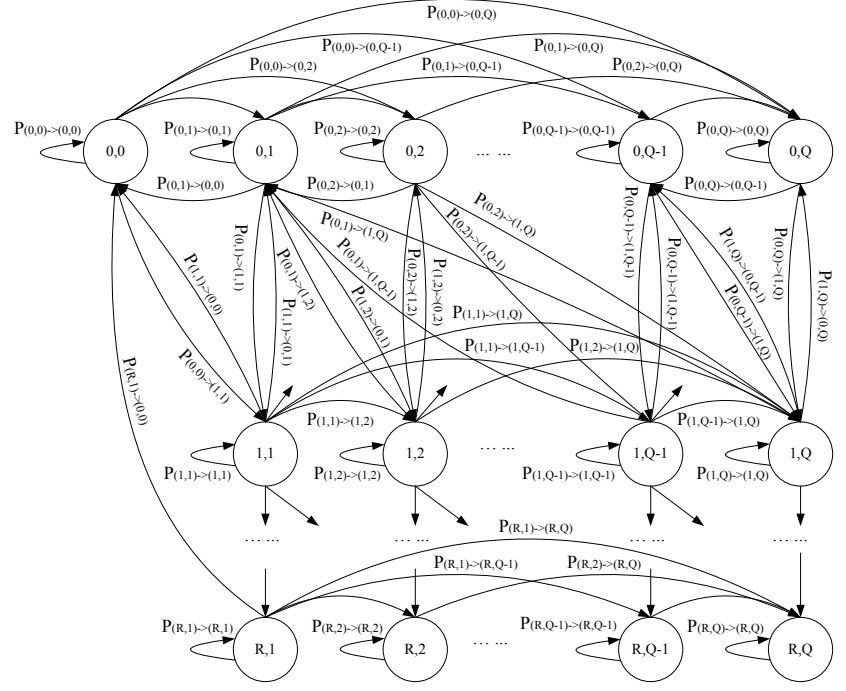

Fig. 3. 2-D Markov model for SMAC with retransmissions.

$P_{(0, j)->(0, Q)}=p_{s} \cdot A_{\geq Q-j+1}+(1-p) \cdot A_{\geq Q-j}, j=1 . . Q$

$P_{(0, j)->(0, j-1)}=p_{s} \cdot A_{0}, j=1 . . Q$

$P_{(i, j)->(i, k)}=(1-p) \cdot A_{k-j}, i=1 . . R, j=1 . . Q-1, k=j . . Q-1$

$P_{(i, j)->(i, Q)}=(1-p) \cdot A_{\geq Q-j}, j=1 . . Q$

Next, we examine the transitions from one retransmission stage to the adjacent higher stage. These transitions correspond to the event that a node has an RTS collision.

$P_{(i, j)->(i+1, k)}=p_{f} \cdot A_{k-j}, i=0 . . R-1, j=1 . . Q-1, k=j . . Q-1$

$P_{(i, j)->(i+1, Q)}=p_{f} \cdot A_{\geq Q-j}, i=0 . . R-1, j=1 . . Q$

Finally, we describe the probabilities of transitions from a non-zero retransmission stage to retransmission stage 0 . These transitions correspond to the events either that a retransmitted DATA packet is successfully delivered, described in (16) and (17), or that a retransmitted DATA packet is discarded due to reaching the retransmission limit, described in (18) and (19).

$P_{(i, j)->(0, k)}=p_{s} \cdot A_{k-j+1}, i=1 . . R-1, j=1 . . Q-1, k=j-1 . . Q-1$ (16)

$P_{(i, j)->(0, Q)}=p_{s} \cdot A_{\geq Q-j+1}, i=1 . . R-1, j=1 . . Q$

$P_{(R, j)->(0, k)}=p \cdot A_{k-j+1}, j=1 . . Q-1, k=j-1 . . Q-1$

$P_{(R, j)->(0, Q)}=p \cdot A_{\geq Q-j+1}, j=1 . . Q, k=j-1 . . Q$

Transitions that are not listed above have a probability of 0 .

The proposed 2-D Markov model assumes that every node has constant $p_{s}$ and $p_{f}$ regardless of any node's queue length or retransmission stage. This assumption is also verified as a good approximation of the real case in [9]-[11] and through our model validation.

\section{THROUGHPUT ANALYSIS}

The throughput of SMAC is defined as the amount of data successfully delivered within a unit time. Since SMAC works in a duty-cycled fashion, the throughput can be calculated within a cycle time. If the proposed Markov model has a unique stationary distribution $\pi$, the throughput of the system

$T_{H R}{ }^{\text {Sys }}=N \cdot\left(1-\pi_{\text {empty } Q}\right) \cdot p_{S} \cdot S / T$ where $\pi_{\text {empty } Q}$ is the stationary probability of the emptyqueue state. Since $N, S$, and $T$ are known, we only need to find $\pi$ and $p_{s}$ for the two proposed Markov models.

\section{A. SMAC without Retransmissions}

The proposed 1-D Markov model with state space $S=\{0,1, \ldots, Q\}$ and transition matrix $P$ has a unique stationary distribution $\pi=\left(\pi_{0}, \ldots, \pi_{Q}\right)$ since the Markov model is irreducible and aperiodic. Therefore,

$\pi_{i} \geq 0$ for any $s_{i} \in S, \sum_{s_{i} \in S} \pi_{i}=1, \pi P=\pi$.

Assuming packet arrival information $\left(\lambda, A_{k}\right.$, and $\left.A_{\geq k}\right)$ is known, the probability of winning the contention $p$ becomes the only variable in the transition matrix $P$. Since $\pi$ is the unique solution for (21), for any $s_{i} \in S, \pi_{i}$ can be represented as a function of $p$. Specifically, let function $f(\cdot)$ describe the relationship between $\pi_{0}$ and $p$, i.e.,

$\pi_{0}=f(p)$

Fig. 4 shows an example of $\pi_{0}=f(p)$ in solid blue. On the one hand, $\pi_{0}$ increases as $p$ increases. When $p$ approaches 0 , a node barely has a chance to transmit a DATA packet, hence $\pi_{0}$ is almost zero. Similarly, when $p$ equals 1 , a node can always win the contention and transmit a DATA packet with no delay. Therefore, $\pi_{0}$ reaches its maximum point. On the other hand, $\pi_{0}$ increases as the duty cycle increases. SMAC with a larger duty cycle has a shorter cycle time. Consequently, the number of packet arrivals in a cycle decreases, and the queue has more chances to be emptied.

Equation (22) depicts the $\left(p, \pi_{0}\right)$ curve that SMAC could be operating on. Also, the assumption that every node has an independent $p$ implies another relationship between $\pi_{0}$ and $p$. Specifically, when a node has a DATA packet to send, the probability that $k$ out of the other $N-1$ nodes are competing for the media $M_{k}$ can be described as a function of $\pi_{0}$.

$M_{k}\left(\pi_{0}\right)=\left(\begin{array}{c}N-1 \\ k\end{array}\right) \cdot\left(1-\pi_{0}\right)^{k} \cdot \pi_{0}^{N-1-k}, k=0 . . N-1$

In the case that $k$ other nodes are competing for the media, the probability of being the winner $p_{k}$ can be calculated as

$p_{k}=\sum_{i=1}^{W} \frac{1}{W} \cdot\left(\frac{W-i+1}{W}\right)^{k}, k=0 . . N-1$

where $W$ is the contention window size. Therefore, let function $g(\cdot)$ describe the relationship between $p$ and $\pi_{0}$,

$p=g\left(\pi_{0}\right)=\sum_{k=0}^{N-1} M_{k}\left(\pi_{0}\right) \cdot p_{k}$.

Fig. 4 shows an example of $p=g\left(\pi_{0}\right)$ in dashed red. When $\pi_{0}=0$, the $N$ nodes are competing for the media every cycle, 


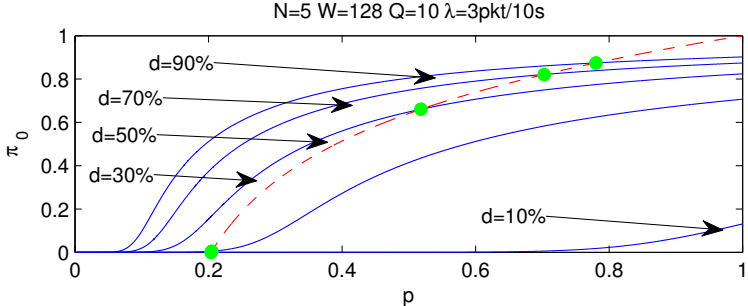

Fig. 4. 1-D Markov model for SMAC without retransmissions.

hence $p$ is around $1 / N$ (the probability of multiple winners of contention is trivial in this case). Moreover, $\pi_{0}=1$ signifies that a node can deliver all the incoming DATA packets without any delay so that no packet will be accumulated in the queue. Hence, $p$ should be 1 .

Solving (22) and (25) allows us to determine the working point of SAMC $\left(p^{*}, \pi_{0}{ }^{*}\right)$, which is also marked in Fig. 4 as the intersection of the solid blue curve and the dashed red curve. For a fixed packet arrival rate, a smaller duty cycle leads to a shorter cycle time, implying fewer nodes on average to compete for the media and fewer packet arrivals in a cycle. Hence, as the duty cycle increases, both $p^{*}$ and $\pi_{0}{ }^{*}$ increase.

$p_{s}$ can be calculated similar to $p$. In the case that a node is competing for the media with $k$ other nodes, the probability of successfully transmitting a DATA packet $p_{s k}$ is

$p_{s k}=\sum_{i=1}^{W} \frac{1}{W} \cdot\left(\frac{W-i}{W}\right)^{k}, k=0 . . N-1$.

Therefore, $p_{s}$ can be represented as a function $h(\cdot)$ of $\pi_{0}$,

$p_{s}=h\left(\pi_{0}\right)=\sum_{k=0}^{N-1} M_{k}\left(\pi_{0}\right) \cdot p_{s k}$.

By substituting $\pi_{\text {empty } Q}=\pi_{0}{ }^{*}$ and $p_{s}{ }^{*}=h\left(\pi_{0}{ }^{*}\right)$ into (20), the throughput of the system can be obtained.

\section{B. SMAC with Retransmissions}

The proposed 2-D Markov model with state space $S=\{(0, j) \mid j=0 . . Q\} \cup\{(i, j) \mid i=1 . . R, j=1 . . Q\} \quad$ and $\quad$ transition matrix $P$ has a unique stationary distribution $\pi$ since the Markov model is irreducible and aperiodic. Therefore,

$\pi_{(i, j)} \geq 0$ for any $s_{(i, j)} \in S, \sum_{s_{(i, j)} \in S} \pi_{(i, j)}=1, \pi P=\pi$.

Assuming packet arrival information $\left(\lambda, A_{k}\right.$, and $\left.A_{\geq k}\right)$ is known, the transition matrix $P$ has two undetermined variables: $p_{s}$ and $p_{f}$. Similar to the case of SMAC without retransmissions, for any $s_{(i, j)} \in S, \pi_{(i, j)}$ can be represented as a function of $p_{s}$ and $p_{f}$. Let function $F(\cdot)$ describe the relationship between $\pi_{(0,0)}$ and $p_{s}, p_{f}$, i.e.,

$\pi_{(0,0)}=F\left(p_{s}, p_{f}\right)$.

Equation (29) determines a surface that SMAC with retransmissions could be working on.

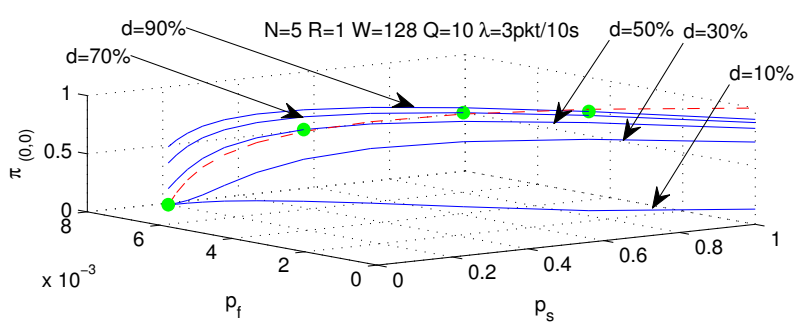

Fig. 5. 2-D Markov model for SMAC with 1 retransmission.

On the other hand, according to (25) and (27), the probability of a DATA packet transmission failure $p_{f}$ can be represented as

$p_{f}=p-p_{s}=g\left(\pi_{(0,0)}\right)-h\left(\pi_{(0,0)}\right)$.

Hence, for every specific $\pi_{(0,0)}$, the corresponding $\left(p_{s}, p_{f}\right)$ is determined by (27) and (30), forming a curve in the space of $p_{s} \times p_{f} \times \pi_{(0,0)}$. Let function $G(\cdot)$ describe the mapping from $\pi_{(0,0)}$ to $\left(p_{s}, p_{f}\right)$,

$\left(p_{s}, p_{f}\right)=G\left(\pi_{(0,0)}\right)=\left(h\left(\pi_{(0,0)}\right), g\left(\pi_{(0,0)}\right)-h\left(\pi_{(0,0)}\right)\right)$.

The curve determined by (31) intersects the surface that is determined by (29). We can solve (29) and (31) to determine the intersection $\left(p_{s}{ }^{*}, p_{f}{ }^{*}, \pi_{(0,0)}{ }^{*}\right)$, which is the working point of SMAC with retransmissions. Substituting $\pi_{\text {empty } Q}=\pi_{(0,0)}{ }^{*}$ and $p_{s}{ }^{*}$ into (20), the throughput of SMAC with retransmissions can be obtained. Fig. 5 shows an example of the 2-D Markov model for SMAC with 1 retransmission under different duty cycles. Equation (31) is shown in dashed red. For the ease of reading the figure, the surface determined by (29) is not completely shown. Instead, the solid blue curves show $\pi_{(0,0)}=F\left(p_{s}, p_{f}\right)$, where $\left(p_{s}, p_{f}\right)$ are in the value domain of (31). Therefore, the intersections of (29) and (31) under different duty cycles are the intersections of the solid blue curves and the dashed red curve. Fig. 5 shows that the working point of SMAC moves to the direction of higher $p_{s}$, lower $p_{f}$, and higher $\pi_{(0,0)}$ when the duty cycle increases.

\section{Model Validation And Discussion}

We validate our Markov model by comparing the analytical throughput obtained from (20) with the throughput obtained from NS-2.32 simulations [12] under various scenarios. We assume (1) nodes are static and are within communication range $(50 \mathrm{~m})$ of each other, (2) each node randomly selects a destination among its neighbors every 200s, (3) DATA arrive at each node according to a Poisson process, (4) MAC layer DATA packet size $S$ is 50 bytes, (5) MAC layer bandwidth is $2 \mathrm{Mbps}$, and (6) nodes have sufficient energy within the 20,000 s simulations. All the simulation points in the figures are the average of 10 runs, and their standard deviations (which are very small) are shown in the figures as well.

\section{A. Varying the Number of Nodes in the Network}

In this experiment, we vary the number of nodes in the 
network from 2 to 30. Fig. 6 shows the system throughput obtained from simulations and our 1-D Markov model for SMAC without retransmissions under different duty cycles. Our analytical results match the simulation results with less than $2.0 \%$ difference in throughput. In Fig. 6, the system throughput increases and then saturates as the number of nodes in the network increases. Before the saturation point, SMAC can transmit all the arriving DATA packets without overflowing the queues. However, when the number of nodes increases to a certain point, SMAC reaches its limit and the queue at each node overflows. As the number of nodes further increases, the system throughput slightly decreases because of a higher probability of an RTS collision. Moreover, SMAC with a higher duty cycle has better saturated system throughput. Since SMAC transmits 1 DATA packet per cycle in the network (neglecting the probability of multiple winners of contention), SMAC with a higher duty cycle has a shorter cycle time and hence a higher system throughput.

We observe that SMAC with and without retransmissions have almost identical throughputs throughout our model validation. In this experiment, our 2-D Markov model with 1 retransmission matches the simulations with less than $2.7 \%$ difference in throughput. To avoid redundancy, we do not show the figures for SMAC with retransmissions but discuss the effect of retransmissions at the end of this section.

\section{B. Varying the Queue Capacity}

In this experiment, we vary the queue capacity at each node from 2 DATA packets to 50 DATA packets. Fig. 7 shows the system throughput obtained from simulations and our 1-D Markov model. Our model again well matches the simulations with less than $2.3 \%$ difference in throughput.

Consider the case that $N=5, W=128, Q=10$, and $\lambda=3 p k t / 10 \mathrm{~s}$ in both Fig. 6 and Fig. 7. Fig. 6 shows that SMAC saturates with $10 \%$ and $30 \%$ duty cycles, but has not yet saturated with $50 \%, 70 \%$ and $90 \%$ duty cycles. Hence from Fig. 7, we can determine that SMAC with 10\% and 30\% duty cycles saturate when $Q \in[2,50]$, and SMAC with $50 \%$, $70 \%$ and $90 \%$ duty cycles have not yet saturated when $Q \in[5,50]$. However, SMAC with $50 \%, 70 \%$ and $90 \%$ duty cycles start to saturate when $Q<5$. Particularly, SMAC with $50 \%$ duty cycle has the largest drop in system throughput when $Q=2$. According to Fig. 6, SMAC with $50 \%$ duty cycle has not yet saturated but approaches the saturation point when

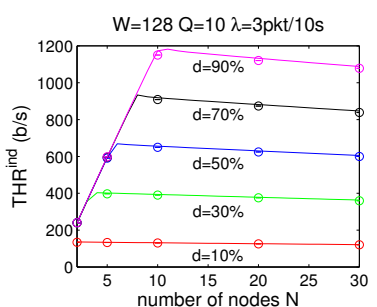

Fig. 6. System throughput of SMAC with different numbers of nodes.

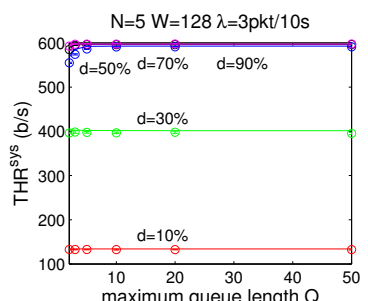

Fig. 7. System throughput of SMAC with different queue capacities.

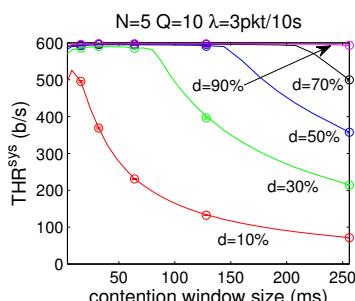

Fig. 8. System throughput of SMAC with different contention window sizes.

$Q=10$. This means that (1) on average, SMAC can keep up with the DATA arrival rate to transmit DATA packets in the network, but (2) the queue should be large enough, e.g., 10 DATA packets in length in this case, to avoid dropping packets due to the jitter of transmission opportunities at each node. A queue with a capacity of 2 DATA packets is too short to smooth the jitter in this scenario. Hence, in Fig. 7 the system throughput of SMAC with 50\% duty cycle drops when $Q=2$. SMAC with $70 \%$ and $90 \%$ duty cycles also experience a slight decrease in their system throughputs with $Q=2$, but since they are further from saturation, the influence of a shorter queue is trivial. In this experiment, our 2-D Markov model with 1 retransmission matches the simulations with less than $2.6 \%$ difference in throughput.

\section{Varying the Contention Window Size}

In this experiment, we vary the contention window size from 4 to 256. Fig. 8 shows the system throughput obtained from simulations and our 1-D Markov model. Again, our model matches the simulations well, with less than $4.8 \%$ difference in throughput. Generally, when SMAC has not yet saturated, the system throughput remains constant. However, as the contention window size increases, the length of a cycle increases and SMAC saturates. Since SMAC can transmit only 1 DATA packet per cycle (neglecting the probability of multiple winners of contention), longer cycle time leads to lower system throughput. On the other hand, when the contention window size is very small, the system throughput has a slight drop. Although decreasing the contention window size leads to a shorter cycle time, which benefits the system throughout, decreasing the window size also increases the probability of multiple winners of contention (probability of an RTS collision), which degrades the system throughput. In this experiment, our 2-D Markov model with 1 retransmission matches the simulations within $1.3 \%$.

\section{Varying the DATA Packet Arrival Rate}

Finally, we vary the DATA packet arrival rate at each node $\lambda$ from $0.2 \mathrm{pkt} / \mathrm{min}$ to $40 \mathrm{pkt} / \mathrm{min}$, corresponding to varying the network traffic load from $6.67 \mathrm{~b} / \mathrm{s}$ to $1,333.33 \mathrm{~b} / \mathrm{s}$. Fig. 9 shows the system throughput obtained from simulations and our 1-D Markov model. Our model once again matches the simulations, with less than $3.9 \%$ difference in throughput. When the traffic load is low, SMAC can transmit DATA packets as fast as they arrive at the network. Hence the system

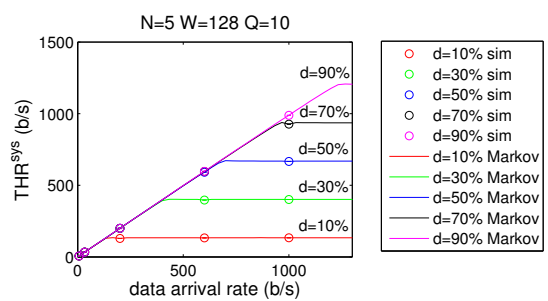

Fig. 9. System throughput of SMAC with different traffic loads. 
throughput increases linearly as the traffic load increases. However, the system throughput remains the same as SMAC saturates. In this experiment, our 2-D Markov model with 1 retransmission matches the simulations within $3.6 \%$.

\section{E. Effect of Retransmissions}

Throughout the model validation, we observe that SMAC with 1 retransmission and SMAC without retransmissions have almost the same performance in term of the system throughput. Retransmitted packets can be considered as extra incoming traffic, arriving at the head of the queue instead of the end of the queue. For a given scenario, if SMAC without retransmissions saturates, the queue at each node overflows. In this case, retransmissions cannot improve the system throughput. On the other hand, for a given scenario, if SMAC without retransmissions has not yet saturated, the system is able to accept some or all of the extra traffic caused by the retransmissions. In this case, SMAC with retransmissions has better system throughput. However, when a network is far from saturation, very few packets experience collisions and need to be retransmitted. Hence, SMAC with retransmissions has almost the same system throughput as SMAC without retransmissions. When a network is close to saturation, the probability of an RTS collision increases and the retransmitted traffic becomes noticeable (but not significant). Hence SMAC with 1 retransmission has slightly higher system throughput. For example, SMAC with $50 \%$ duty cycle approaches saturation at traffic load 600b/s in Fig. 9. At this point, SMAC with 1 retransmission has $1.4 \%$ higher throughput than SMAC without retransmissions. As the number of retransmissions increases, the improvement can reach up to $1.5 \%{ }^{1}$

\section{RELATED WORK}

Although no existing work evaluates the performance of SMAC analytically, there has been previous work utilizing a Markov model to describe the behavior of a MAC protocol. Specifically, Bianchi proposed a Markov model to analyze the saturation throughput of IEEE 802.11 with unlimited retransmissions [9]. Wu et al. modified Bianchi's model to support finite retransmissions [10]. Robinson and Randhawa extended Bianchi's model to analyze the saturation throughput of IEEE 802.11e [13]. Later, Liaw, Dadej, and Jayasuriya extended Bianchi's model to analyze unsaturated throughput of IEEE 802.11 [11]. However, all of these Markov models assumed that a node has an infinite queue, which is not true in reality. Ghaboosi, Khalaj, Xiao, and Latva-aho proposed a parallel space-time Markov chain, which modeled the queue status at each node for IEEE 802.11 networks [7]. Meanwhile, Fallah et al. [8] and He et al. [14] also proposed Markov models to analyze the throughput of IEEE 802.16 and IEEE 802.15.4 networks, respectively. Recently, Shi, Aryafar,

\footnotetext{
${ }^{1}$ This result is obtained by comparing the analytical throughput of SMAC without retransmissions to the analytical throughput of SMAC with unlimited retransmissions, using a modified 1-D Markov model by changing $p$ to $p_{s}$ in (1)-(6). This result is also validated by simulations.
}

Sakinidis and Kinghtly proposed a Markov model to evaluate the fairness of synchronized CSMA [15]. However, their model does not consider queue capacity and data arrival rate.

\section{CONCLUSION AND FUTURE WORK}

In this paper, we modeled and analyzed the throughput of SMAC with finite queue capacity. Specifically, a 1-D Markov model is proposed to describe the behavior of SMAC without retransmissions, and a 2-D Markov model is used to describe the behavior of SMAC with retransmissions. The throughput of SMAC can be obtained from the proposed model, and the results are validated by comprehensive simulations. Our Markov model and throughput analysis can be used to estimate the performance of SMAC, optimize the SMAC parameters, and optimize the duty cycle to arbitrate the tradeoff between throughput and network lifetime. Our future work includes delay analysis based on the proposed Markov model and performance analysis for the case of non-ideal channels.

\section{ACKNOWLEDGMENT}

This work was supported in part by the NSF \#CNS0448046.

\section{REFERENCES}

[1] H. Luo, J. Luo, Y. Liu, and S. K. Das, "Adaptive data fusion for energy efficient routing in wireless sensor networks", IEEE Trans. Computers, vol. 55 , no. 10 , Oct. 2006, pp. 1286-1298.

[2] W. Ye, J. Heidemann, and D. Estrin, "An energy-efficient MAC protocol for wireless sensor networks", in Proc. INFOCOM'02, 2002, pp. 1567-1576.

[3] T. Dam and K. Langendoen, "An adaptive energy-efficient MAC protocol for wireless sensor networks", in Proc. Sensys'03, 2003, pp. 171-180.

[4] J. Polastre, J. Hill and D. Culler, "Versatile low power media access for wireless sensor networks", in Proc. Sensys'04, 2004, pp. 95-107.

[5] K. Kumar and P. Kumar, "Tmote implementation of BMAC and SMAC protocols," $h$ ttp://www.cse.iitk.ac.in/users/vkirankr/wireless_report.pdf.

[6] V. Tippanagoudar, I. Mahgoub, and A. Badi, "Implementation of the Sensor-MAC protocol for the JiST/SWANS simulator", in Proc. AICCSA'07, 2007, pp. 225-232.

[7] K. Ghaboosi, B. H. Khalaj, Y. Xiao, and M. Latva-aho, "Modeling IEEE 802.11 DCF using parallel space-time Markov chain", IEEE Trans. Vehicular Technology, vol. 57, no. 4, Jul. 2008, pp. 2404-2413.

[8] Y. Fallah, F. Agharebparast, M. Minhas, H. Alnuweiri, and V. Leung, "Analytical modeling of contention-based bandwidth request mechanism in IEEE 802.16 wireless networks", IEEE Trans. Vehicular Technology, vol. 57, no.5, Sep. 2008, pp. 3094-3106.

[9] G. Bianchi, "IEEE 802.11 - saturation throughput analysis", IEEE Communication Letters, vol. 1, no. 12, Dec. 1998, pp. 318-320.

[10] H. Wu, Y. Peng, K. Long, S. Cheng, and J. Ma, "Performance of reliable transport protocol over IEEE 802.11 wireless LAN: analysis and enhancement”, in Proc. INFOCOM'02, 2002, pp. 599-607.

[11] Y. S. Liaw, A. Dadej, and A. Jayasuriya, "Performance analysis of IEEE 802.11 DCF under limited load", in Proc. APCC'05, 2005, pp. 759-763.

[12] http://www.isi.edu/nsnam/ns/

[13] J. W. Robinson, T. S. Randhawa, "Saturation throughput analysis of IEEE 802.11e enhanced distributed coordination function", IEEE JSAC, vol. 22, no. 5, Jun. 2004, pp. 917-928.

[14] J. He, Z. Tang, H. Chen, and Q. Zhang, "An accurate and scalable analytical model for IEEE 802.15.4 slotted CSMA/CA networks", IEEE Trans. Wireless Communications, vol. 8, no. 1, Jan. 2009, pp. 440-448.

[15] J. Shi, E. Aryafar, T. Salonidis, and E. Knightly, "Synchronized CSMA contention: model, implementation and evaluation", in Proc. INFOCOM'09, 2009, pp. 2052-2060. 